\title{
Handover Algorithm based VLP using Mobility Prediction Database for Vehicular Network
}

\author{
Arfah A. Hasbollah ${ }^{1}$, Sharifah H. S. Ariffin ${ }^{2}$, Nurzal E. Ghazali ${ }^{3}$, K. Mohamad Yusuf ${ }^{4}$, \\ Hiroaki Morino ${ }^{5}$ \\ ${ }^{1}$ Department of Electrical Engineering, Politeknik Ibrahim Sultan, Malaysia \\ ${ }^{2,3,4}$ Faculty of Electrical Engineering, Universiti Teknologi Malaysia, Malaysia \\ ${ }^{5}$ Department of Communications Engineering, Shibaura Institute Technology, Japan
}

\begin{tabular}{l}
\hline \hline Article Info \\
\hline Article history: \\
Received Feb 20, 2018 \\
Revised May 30, 2018 \\
Accepted Jun 17, 2018 \\
\hline
\end{tabular}

\section{Keyword:}

Database

Handover algorithm

Handover delay

Mobility prediction

Vehicular network

\begin{abstract}
This paper proposes an improved handover algorithm method for vehicle location prediction (VLP-HA) using mobility prediction database. The main advantage of this method is the mobility prediction database is based on real traffic data traces. Furthermore, the proposed method has the ability to reduce handover decision time and solve resource allocation problem. The algorithm is simple and can be computed very rapidly; thus, its implementation for a high-speed vehicle is possible. To evaluate the effectiveness of the proposed method, QualNet simulation is carried out under different velocity scenarios. Its performance is compared with conventional handover method. The superiority of the proposed method over conventional handover method in deciding the best handover location and choosing candidate access points is highlighted by simulation. It was found that VLP-HA has clearly reduced handover delay by $45 \%$ compared to handover without VLP, give high accuracy, hence low complexity algorithm.
\end{abstract}

Copyright $\odot 2018$ Institute of Advanced Engineering and Science. All rights reserved.

\section{Corresponding Author:}

Arfah binti Ahmad Hasbollah,

Department of Electrical Engineering,

Politeknik Ibrahim Sultan,

KM 10, Jalan Kong Kong, 81700 Pasir Gudang, Johor Darul Takzim, Malaysia.

Email: arfah@fkegraduate.utm.my

\section{INTRODUCTION}

The number of mobile users connected through wireless networks has significantly increased in recent years due to the increasing number of wireless device deployments, which open the way to ubiquitous networks connectivity. Handover service is critical to ensure communication service is well maintained along the journey. Due to the mobility nature of the mobile user, low latency reliable communication between end users is hard to achieve. In order to maintain the quality of service (QoS) and to provide large coverage, robust and reliable handover process should be developed. Current studies on handover algorithm of the mobile user have focused on the decision making and resource allocation [1]-[10]. However, for vehicular user mobility, the task to maintain seamless network is very challenging. The wireless connection between vehicle access problems especially in high-speed mobile scenarios such as frequent handover which lead to frequent cell selection, higher end-to-end delay, call drop and inaccessibility data service [11].

One way to overcome this problem is by using prediction method for allocating appropriate resources and allocate in the shortest time for handover of the mobile user. For example, [12] showed that people moved from one location to another can be predicted using commercial mobile telecoms network and more recently, [13] using Kalman filter to predict future vehicle's location from vehicles traces collected under the different situation. Even though the prediction method was demonstrated years ago, little attention has been paid to the appropriate access point (AP) selection for handover decision making. 
It is noted that the technical details and system procedures of handover in heterogeneous networks fifth generation $(5 \mathrm{G})$ attribute system have fundamental differences with other systems, however, there is no specification that has been designed or released. As the main contributions of this paper, based on the frame structures, protocol, measurement procedure; the system information and handover procedure are analyzed and designed on the basis of the LTE-Advanced network. Thus, to allow the predictability and generality of this network architecture to be maintained. The key of research of this paper, the details of the handover procedure which includes the new handover decision-making scheme and signaling flow during intra-macro cell and inter-macro cell handover are presented and thoroughly designed.

The paper presents a set of the prediction method for selecting the best AP. On the basis of this method, it then describes the preparation of handover algorithm. The combination of these two methods will provide an effective technique to improve handover performance on vehicular networks. The remainder of this paper is structured as follows. Section 2 presents related work. Section 3 presents the mobility prediction proposed scheme. Section 4 portrays the handover algorithm combined with mobility prediction, simulation setting and contributed scenarios. Section 5 presents and discusses the simulation results and finally, the paper concludes in Section 6.

\section{RESEARCH METHOD}

The mobility prediction can enhance the handover performance by reducing handover latency, packet jitter, and end-to-end delay. One key approach for mobility prediction is using Markov Chain predictor. In this section, a mobility prediction via Markov Chain as a technique to assist the handover procedure in the vehicular network is proposed. The overview of envisions about the vehicles network data available and the system network architecture is explained first before describing the prediction algorithm. Wireless network for the user in vehicles that traveling from one cell to another is assumed to have open access mode. Every node or AP records its coordinates as well as its ID number. Besides, there is a centralized approach to collect the history of every node and execute the prediction algorithm. Therefore, the trajectory of the vehicles is denoted by sequences of AP ID numbers, which form a model for prediction scheming.

Markov Chain predictor is based on probability matrix that utilizes trajectory of the users that are stored in a database. The database consists of multiple available sources of information and is organized in the proposed data structure. In this work, the database is collected from user's data traces using logging report, which are then processed into transaction database. The Markov chain equation can be derived as:

$$
p_{n}=p_{t}[P]^{n}
$$

Where,

$$
\begin{aligned}
& p_{t}=\text { initial distribution matrix }, \\
& P=\text { current transition probability matrix, } \\
& n=\text { number of state transition. }
\end{aligned}
$$

\subsection{User mobility pattern}

Equation (1) is used to predict vehicle's next location. The main parameter for this equation is TPM. Many studies have shown that value in TPM is trained from user's mobility history [13], [14]. The collection of user's mobile history provides useful information such as frequently visited locations, common routes and received the radio signal. However, it is not easy to deal with such data. The user's mobility history may consume much memory, energy, and bandwidth, especially at the base stations that are frequently visited. Therefore, the data should be checked and pre-processed before carrying the analysis. A method called data mining is needed to extract and analyze data. In this section, the formal definitions to model mobility pattern and rules are given.

Definition 1. Let $C$ and $Y=T$ as a set of access point ID and time respectively. The ordered pairs $p=(c, t)$, where $c \in C$ and $t \in T$, is called a point. Denote $P$ to be the set of all points $P=C \times T=\{(c, t) \mid c \in C$ and $t \in T\}$.

Two points $p_{1}=\left(c_{1}, t_{1}\right)$ and $p_{2}=\left(c_{2}, t_{2}\right)$ are considered. Point $p_{1}$ is equivalent to $p_{2}$, if and only if $c_{1}=c_{2}$ and $t_{1}=t_{2}$. If $t_{1}<t_{2}$, point $p_{1}$ is defined as an earlier location than point $p_{2}$ which means the user connected to point $p_{1}$ before connected to point $p_{2}$.

Definition 2. A trajectory of the user is defined as a finite sequence of the points $p\left(i . e . p_{1}, p_{2}, \ldots p_{k}\right)$ in $C \times T$ space where point $p_{i}=\left(c_{i}, t_{i}\right)$ for $1 \leq i \leq k$. 
The value of point $\mathrm{p}$ must be unique in the trajectory for each user (e.g. $p_{1} \neq p_{2}$ ), where the user cannot be connected to the same AP at the same time. The value of time $t$ shall be unique in the trajectory. However, the value of AP ID $c$ can be the same with different time, $t$. Means, the user can be connected to the same AP, but at a different time. If there is a case where point $p_{i}$ is the same as other point $p$, the data obtained is set as an information error. The earlier information is stored while other duplicate information shall be removed from the log file.

Definition 3. Time interval $t_{i}$ is an interval time between two sequence points $p\left(i\right.$. e. $_{i}$ and $\left.p_{i+1}\right)$. Time gap $t_{g}$ is a maximum interval time that the user connected to a point $p$. If the time interval $t_{i} \geq$ time gap $t_{g}$, the user is assumed to stop moving or reach its destination.

The time gap $t_{g}$ is introduced to create a partition between trajectories and identify the last destination of the user. The accuracy of the transactional database is expected to be increased when the time gap $t_{g}$ is used. The value of the time gap $t_{g}$ should be chosen properly because it may affect the prediction accuracy of the user's next location.

From the log file, the source-destination table is computed then the transactional database is developed. The transactional database shows a relationship between the source AP and the destination AP. This is the final step of the user's mobility history scheme before the value of the transactional database is utilized in the Markov Chain equation to predict the user's next location.

\subsection{Transactional database}

The first step in data mining process is to create a log file as in Figure 1. The format of the log file is inspired by [15]. Data contained in the log file are a date, time, location, and type of transport. The date represents a date the user connect to the AP. Location represents AP ID number the user is connected at a particular time. Time represents the time the user is connected to the particular AP. And lastly, transport represents what kind of transport they use at the particular time because different vehicles have different speed. This log file will be updated each time user moves from one location to another. Data collection is done by considering some assumption: 1) Wireless network in campus is open access mode. 2) All APs are located in every building.

\begin{tabular}{|c|c|c|c|c|}
\hline Date & Time (am/pm) & $\begin{array}{c}\text { Location (building or } \\
\text { the nearest, i.e. P19, } \\
\text { P04, D06) }\end{array}$ & $\begin{array}{c}\text { Duration } \\
\text { spent }\end{array}$ & $\begin{array}{c}\text { Type of transport (car, } \\
\text { motorcycle, cycle, or } \\
\text { walk) }\end{array}$ \\
\hline
\end{tabular}

Figure 1. Log file

Data collection must be done when the mobile client is in 'idle' mode and not communicating with any AP. The idle mode is chosen because to avoid the effect on actual data performance. In this research, a group of real vehicles user mobility traces in Universiti Teknologi Malaysia (UTM) are used which consists of undergraduate students, post-graduate students, and staff. With our pre-process on the original data, the mobility trace denotes associated history of each vehicle by the cell number in the network under a simple mobility model assumption. After logging report is acquired, the transactional database is created to relate between the source and destination base station. The most frequent visited base station will be detected via the database. Figure 2 shows the step of transactional database development.

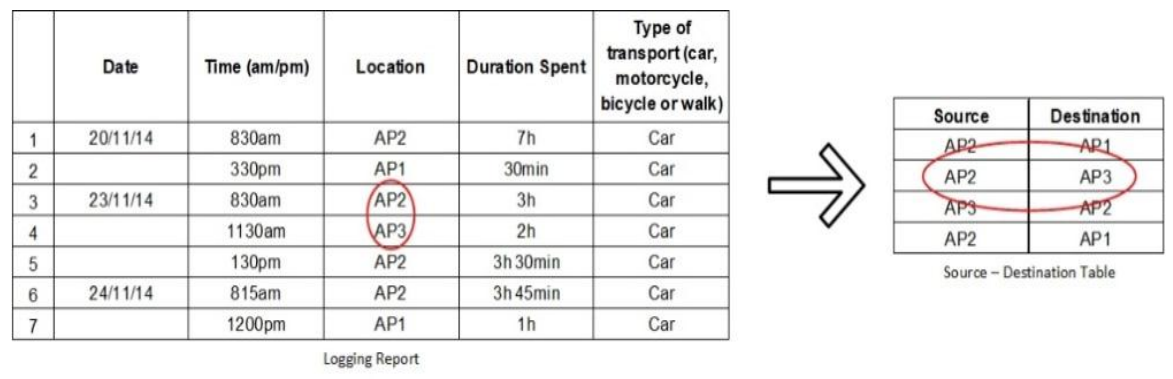

Figure 2. Source-destination table 
First, logging report is converted into the source-destination table to identify user's trajectory. From the source-destination table, a transactional database is developed based on the relationship between source and destination AP. Day and time should be considered when converting logging report into the sourcedestination table. The starting location for each day is set as the source, and then next location will become the destination. If there is third movement after that, the current location will become the source and next location will become the destination. These steps will be carried out continuously throughout the day according to logging report. The last location for that day will become the destination. Then for the next day location, it will become the new source.

From the source-destination table, the transactional database is developed base on the relationship between source and destination AP. The first column in the transactional database represents source AP and the first row represents destination AP. The value of each relationship determines the frequency of user attached to each AP. The grand total is the total amount of user's transition from each base station to another. Once the transactional database is created, the TPM is generated.

TPM can be defined by dividing each value by the total value for each row in the transactional database. The value of transition probability is verified using summation for each row in the transactional database that should equal to 1. TPM value can be derived following Equation (2). This will be applied to all value in the transactional database. This process will occur continuously along with the acquired data. Then, this TPM used in Markov equation to predict vehicle's next location. After mobility prediction is calculated, prediction accuracy is computed for proper estimation of user's next location. The predicted result will be verified to lessen any error in prediction. Figure 3 shows the step of generating TPM from the transactional database.

$$
T P M=\frac{p_{i+1}}{\Sigma p_{i+1}}
$$

Where $p_{i}+1=$ number of destination.

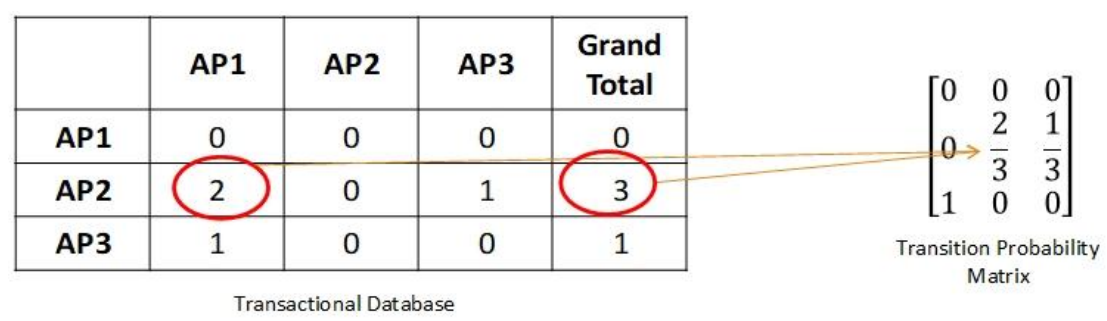

Figure 3. Step for generating TPM

The mobility prediction can be calculated after determining the initial probability or initial state of the mobile user. As mention before, initial state can be calculated by some parameter such as the number of the user, velocity of mobile user or distance target, or location, etc. This parameter is presented as initial state matrix. Furthermore, by using user's initial state or GPS location and velocity as the value of initial state matrix and TPM as Equation (1), the next location of the mobile user can be calculated.

The predicted result then checked to mitigate an error in prediction. Ideal case in handover is mobile user should handoff to the adjacent AP. However, if predicted AP is not adjacent to the previous AP, the predicted AP is considered an error. In order to verify the efficiency of mobility prediction technique, prediction accuracy is calculated based on the ratio between the number of correct prediction and the total number of predictions as in Equation (3). Flowchart of proposed mobility prediction process is shown in Figure 5.

$$
\operatorname{Pr} \text { ediction accuracy }=\frac{\sum N_{c p}}{\sum N_{p}}
$$

Where $N_{c p}=$ number of correct predictions and $N_{p}=$ number of predictions. Figure 4 illustrate the performance comparisons of VLP method and heuristic method [13]. Simulation result demonstrates that the 
proposed predictive mobility scheme outperformed a heuristic predictive scheme because VLP method achieves higher prediction accuracy which peaks at about 0.96 compared to the heuristic method which is peaks at about 0.71 .

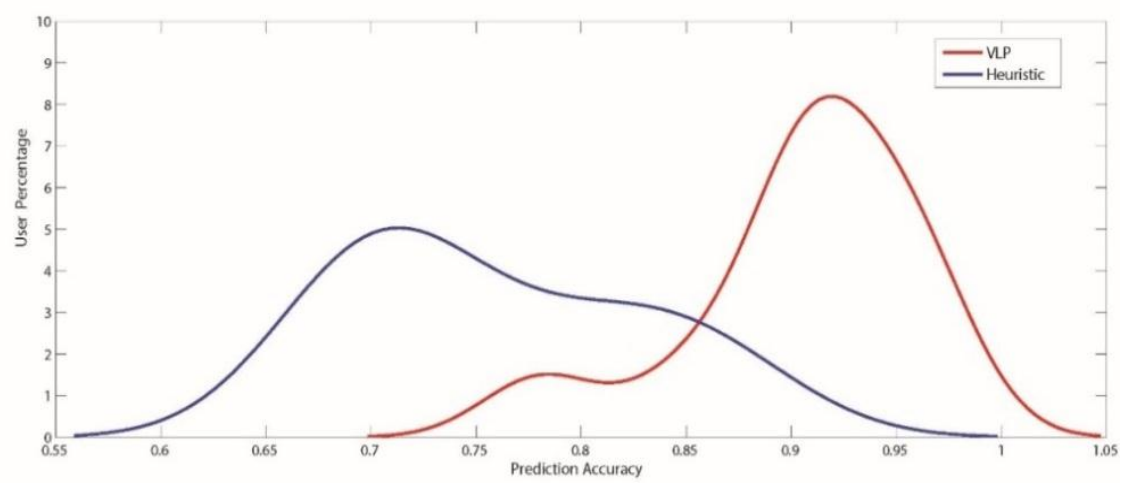

Figure 4. Prediction accuracy comparison between VLP method and heuristic method

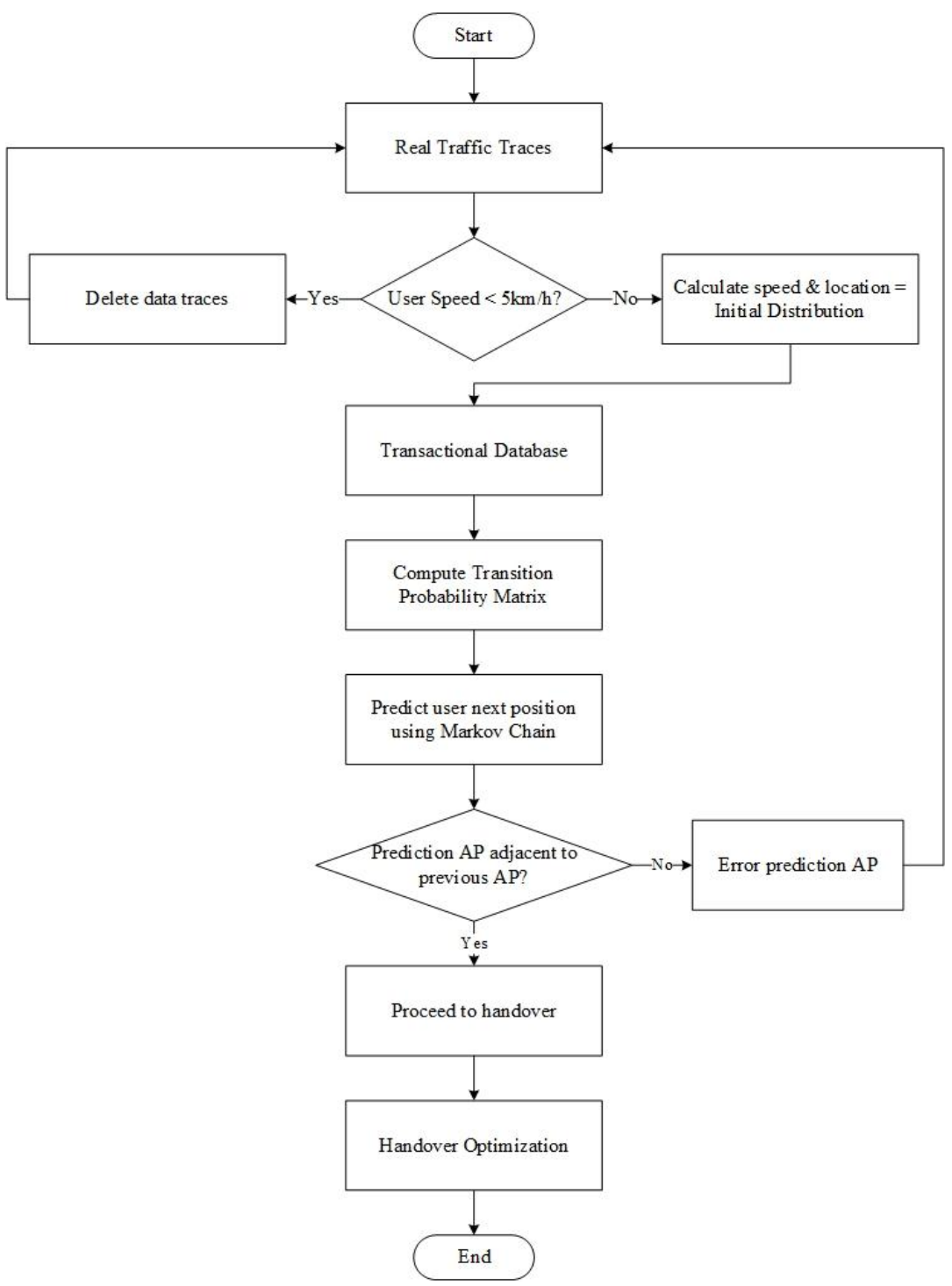

Figure 5. VLP technique 


\section{HANDOVER ALGORITHM FOR VEHICULAR NETWORK}

According to [16], a conventional handover procedure consists initiation step, network selection step, and execution step. In initiation step, UE measures the channel qualities such as Received Signal Strength (RSS), Signal to Interference Ratio (SIR), distance and others from a different cell. Then UE sends the measurement report back to the serving cell. The serving cell then forwards the measurement report to control unit. Control unit decides which cell is the best to maintain the connection in network selection step. At this rate, the handover decision-making process will be performed based on handover threshold. If the handover is required, then control unit informs the target cell and lets serving cell send the handover command to UE. Finally, in execution step, the UE starts to set up a connection with the target cell by sending authentication, synchronization, network reconfiguration, etc. After the handover process is completed, UE sends an indication signal to the target cell. From these procedures, it involves many wireless and backbone exchanging signal. It may take some time to complete the handover. In this section, a handover algorithm that overcomes this problem is delivered.

The proposed vehicular location prediction handover algorithm (VLP_HA) was designed by adopting Markov chain mobility prediction feature, was a promising technique to improve the overall wireless network performance. This improvement was important to minimize handover delay and to improve data throughput. As explained in the previous section, handover decision phase is the important part to ensure the mobile user has good communication quality. VLP_HA requires the prediction results from the proposed vehicle location prediction (VLP) to assist the handover decision.

VLP_HA is started by the serving cell receives the measurement report from UE. Control unit then received the measurement report and decided the target cell by examining the neighbor cell with highest RSS value. At the same time, the best neighbor cell was verified with the prediction results. If the best neighbor cell was same as the predicted cell, it suggested that the target cell is the location that UE should go. Thus, the handover was needed. However, if the best neighbor cell was not same as predicted cell, it assumed that the target cell was not the location that UE should go. Therefore, the handover was unnecessary and it will be delayed for a while. The handover can be performed when the target cell RSS is higher than source cell RSS at least by handover trigger offset. This procedure was conducted to minimize the number of unnecessary handovers that lead to ping-pong effect. Moreover, VLP_HA was a simple algorithm and could achieve fast handover. Since the coverage area of AP is small, UE may pass through the coverage area within the short time, which is why fast handover was needed.

The network simulation scenarios are set as shown in Table 1. The simulation time was set for 100 seconds to observe the overall network performance as well as the arrival time for UE. All APs have the same characteristic which transmits $30 \mathrm{dBm}$ powers. The scenario considers the open access mode for all APs. Hence, the UE can connect to the APs without any restriction.

The handover simulations were performed using QualNet to observe the handover performance when the UE speed is varied. Each simulation result was recorded, tabulated and the handover performance was analyzed. The network simulation scenario is based on real topology in UTM within the area of $1.5 \mathrm{~km}$ $1.5 \mathrm{~km}$ as shown in Figure 6. This network scenario consists a group of wireless APs organized for 1 mobile user. It also consists 4 mobility management entity (MME) and serving gateway (SGW) nodes supporting to the AP region and a core network. SGW shall forward the data that come from the base station and packet data network gateway. MME is used to control the high-level operation such as choosing the right SGW for the UE and authenticating them. MME also responsible for controlling the mobility between the LTE networks with other access networks.

As simulation starts, UE moves toward the destination. As it approaches next eNB, UE starts to lose network coverage. UE will start sending the measurement report to next eNB to start handover process. However, the challenge here is eNBs is closed with each other and mobile UE is not allowed UE to do normal handover process. Ping pong effect also happened at this stage. But with predict handover scheme, the next eNB is predicted and handover process time will be shortened because handover decision is taken based on predicted next eNB.

Table 1. Simulation parameter

\begin{tabular}{cc}
\hline Variable & Value \\
\hline Simulation Time & $100 \mathrm{~s}$ \\
Transmission Power & $30 \mathrm{dBm}$ \\
Number of UE & 1 \\
UE velocity & $1-10 \mathrm{~m} / \mathrm{s}$ \\
Receiver Sensitivity & $-80 \mathrm{dBm}$ \\
Handover RSS Trigger & $-72 \mathrm{~dB}$ \\
\hline
\end{tabular}

Int J Elec \& Comp Eng, Vol. 8, No. 4, August 2018 : 2477 - 2485 


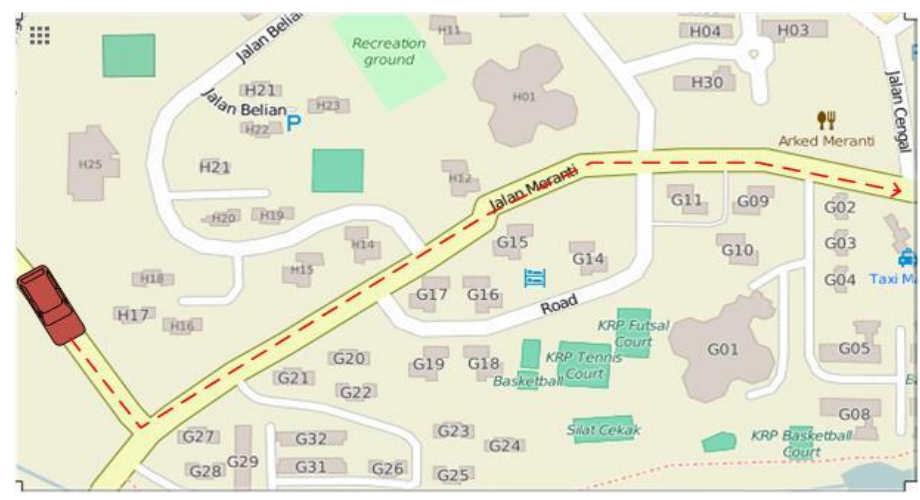

Figure 6. Network simulation scenario

\section{RESULT AND DISCUSSION}

This section analyzed the simulation result of the handover performance and discussed in detail. The network performances were measured in term of delivery delay, network throughput, and packet delivery ratio. Delivery delay performance is to look at the delivery delay rate when data was being sent to AP during the communication. If the delivery delay rate is high, it means that the network is in the congested state. A jitter arriving, caused by network congestion, timing drifts, or route changes. So, delivery delay plays an important role to determine the network jitter as the lower delivery rate, the less network jitter occurs. Meanwhile, the network throughput is the sum of the data rates that are delivered to all eNBs nodes or APs in a network. Packet delivery ratio is the ratio between the number of data packets sent by source nodes and the number of packets received by the destination.

Figure 7 depicts the delivery delay for user speed between $1 \mathrm{~m} / \mathrm{s}$ to $10 \mathrm{~m} / \mathrm{s}$. Compared to conventional handover, network delay rate for predicted handover scheme is improved by $45 \%$ which is indicates APs trigger handover process is faster than conventional handover scheme. Additionally, knowing where destination nodes are heading with high accuracy, packets forwarders can make better decisions to choose the next hop. It could be concluded that VLP-HA can perform faster handover compared to conventional handover.

Figure 8 shows the handover throughput versus user's speed $(1-10 \mathrm{~m} / \mathrm{s})$. In the simulation scenario, UE is moving across the network on a predefined path, and the throughput would fluctuate when the UE is moving into the cell center or moving out to the cell edge. According to Figure 8, the network throughput seems to be increased by $4 \%$ for predicted handover scheme greater than conventional handover scheme. This concludes that mobility prediction can significantly and stably improve the capacity of users in the vehicle, thus providing the possibility to support high data rate services.

Figure 9 plots the packet delivery ratio. It turns out that the packet delivery ratio (PDR) is almost same for conventional handover and predicted handover. It means that the ratio between two schemes is almost identical and not much difference. PDR for VLP-HA have difference about $1 \%$ with conventional handover. Apparently, it also depends on the UE speed. The PDR starts with high value. However, when the UE speed increases, the PDR drops to below $85 \%$ due to the packet dropping over frequent handover. Although network throughput for predicted handover is higher than conventional handover, the higher UE predictive state information in PDR offers better performance.

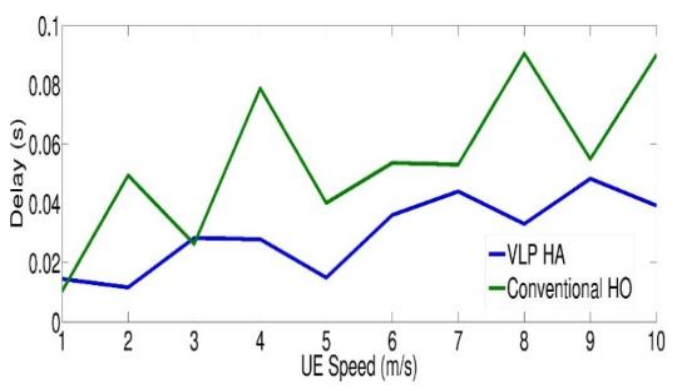

Figure 7. Handover delay with and without mobility predictions under different velocities 


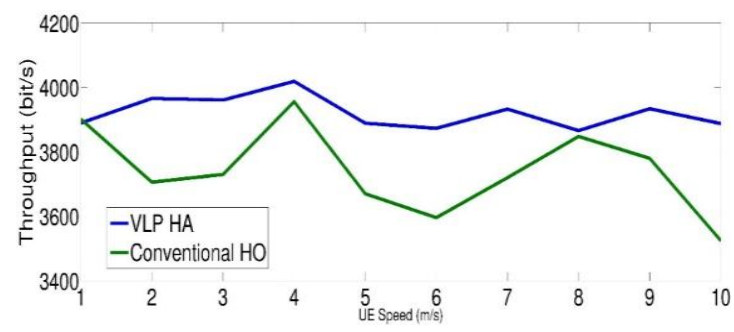

Figure 8. Handover throughput with and without mobility predictions

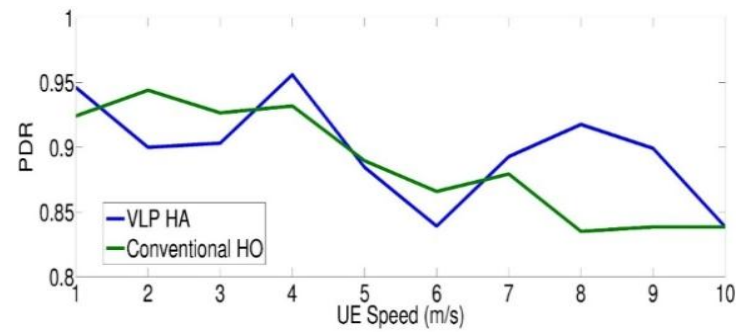

Figure 9. Packet delivery ratio when under different velocities

\section{CONCLUSION}

In this paper, the handover with mobility prediction algorithm is used in the vehicular network. It is shown that the conventional handover techniques take a lot of time from initiation step to network selection and execution step. Hence, it will be the disadvantage for the mobile user which leads to frequent cell selection, higher end-to-end delay, call drop and inaccessibility data service. To improve the handover method, a simple and efficient method is proposed to allocate the network resource for the shortest time which is using mobility prediction. The results indicate that the proposed handover method outperforms the conventional handover method and gives several advantages: 1) It has lower end-to-end delay; 2) it exhibits more data throughput within the network; 3) the mobility prediction algorithm can be easily developed using user's history database; 4) The mobility prediction have high accuracy, hence give low complexity algorithm.

\section{ACKNOWLEDGEMENTS}

The authors would like to thank all those had contributed towards making this research successful, especially the support from research team Advanced Telecommunication Technology (ATT) and Shibaura Institute of Technology (SIT). The authors wish to express their gratitude to the Ministry of Higher Education Malaysia (MOHE), Research Management Center UTM (RMC) for the sponsorship and financial support (Vot Number Q.J13000.2523.13H24 ).

\section{REFERENCES}

[1] H. Mahajan, Payal; Singh, Kuldeep; Kaur, "Handover decision mechanism in interworking technologies using radial basis functions," IAES Int. J. Artif. Intell., vol. 3, no. 2, pp. 79-83, 2014.

[2] M. Mouton, G. Castignani, R. Frank, and T. Engel, "Enabling Vehicular Mobility in City-wide IEEE 802.11 Networks Through Predictive Handovers," Veh. Commun., vol. 2, no. 2, pp. 59-69, 2015.

[3] A. S. Sadiq, K. A. Bakar, K. Z. Ghafoor, and A. J. Gonzalez, "Mobility and Signal Strength-Aware Handover Decision in Mobile IPv6 based Wireless LAN," in International MultiConference of Engineers and Computer Scientists, 2011.

[4] W. Xiaonan and Q. Huanyan, "Mobility Management Solution for IPv6-based Vehicular Networks," Comput. Stand. Interfaces, vol. 36, no. 1, pp. 66-75, 2013.

[5] J. Moon and D. Cho, "Efficient Group Handoff Decision Algorithm for Wireless Networks with Mobile Access Points," in 2011 - MILCOM 2011 Military Communications Conference, 2011, pp. 1096-1101.

[6] X. Xu, "Access Control and Handover Strategy for Multiple Access Points Cooperative Communication in Vehicle Environments," in Vehicular Technologies - Deployment and Applications, InTech, 2013, pp. 97-115.

[7] S. Chae, T. Nguyen, and Y. M. Jang, "Seamless QoS-Enabled Handover Scheme Using CoMP in Fast Moving Vehicular Networks," Int. J. Distrib. Sens. Networks, pp. 1-8, 2013. 
[8] D. Barth, S. Bellahsene, and L. Kloul, "Mobility Prediction Using Mobile User Profiles," in 2011 IEEE 19th Annual International Symposium on Modelling, Analysis, and Simulation of Computer and Telecommunication Systems, 2011, pp. 286-294.

[9] G. Zhang and F. Liu, "An Auction Approach to Group Handover with Mobility Prediction in Heterogeneous Vehicular Networks," in 2011 11th International Conference on ITS Telecommunications, 2011, pp. 584-589.

[10] L. Zhanjun, M. Qichao, R. Cong, and C. Qianbin, "Load Balancing Based on the Specific Offset of Handover," TELKOMNIKA (Telecommunication Computing, Electronics and Control), vol. 12, no. 8, pp. 6281-6290, 2014.

[11] R. Zhang, M. Wu, Y. Zhang, and L. Luan, "Alternative Reference Point Based Handover Algorithm for LTE Highspeed Rail,” Indones. J. Electr. Eng. Comput. Sci., vol. 12, no. 3, pp. 2278-2284, 2014.

[12] G. Xue, Y. Luo, J. Yu, and M. Li, "A Novel Vehicular Location Prediction Based On Mobility Patterns for Routing In Urban VANET," EURASIP J. Wirel. Commun. Netw., no. 1, p. 222, 2012.

[13] N. A. Amirrudin, S. H. S. Ariffin, N. N. N. A. Malik, and N. E. Ghazali, "User's Mobility History-based Mobility Prediction in LTE Femtocells Network," in IEEE International RF and Microwave Conference (RFM2013), 2013, pp. 105-110.

[14] S. Chen, Y. Li, W. Ren, D. Jin, and P. Hui, "Location Prediction for Large Scale Urban Vehicular Mobility," in Wireless Communications and Mobile Computing Conference (IWCMC), 2013, pp. 1733-1737.

[15] N. A. Amirrudin, "Mobility Prediction via Markov Model in LTE Femtocell," Int. J. Comput. Appl., vol. 65, no. 18, pp. 40-44, 2013.

[16] Q.-A. Zeng and D. P. Agrawal, "Handoff in Wireless Mobile Networks," in Handbook of Wireless Networks and Mobile Computing, I. Stojmenovic, Ed. John Wiley \& Sons, 2002, p. 662.

\section{BIOGRAPHIES OF AUTHORS}

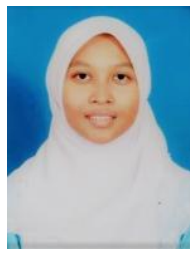

Arfah A. Hasbollah received the B.E degree in Electrical (Electronics) in 2007 and M.E. degree in Electrical-Electronics and Telecommunication in 2009 from Universiti Teknologi Malaysia (UTM). Now she is pursuing Ph.D in Universiti Teknologi Malaysia. She is currently an Officer of Higher Education in Politeknik Ibrahim Sultan. Her current research interests include vehicular network, mobility management in LTE, LTE-A and 5G, and Mobile Computing System.

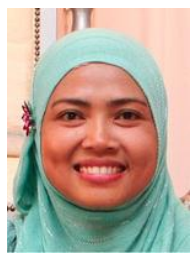

Sharifah Hafizah Sed Ariffin received her B.Eng (Hons) from London Metropolitan University (formerly known as University of North London) in 1997, and obtained her MEE and Ph.D in 2001 and 2006 from Universiti Teknologi Malaysia, and Queen Mary, University of London, London respectively. She is currently Associate Professor with Faculty of Electrical Engineering, Universiti Teknologi Malaysia. Her current research interests are in Internet of Things, Ubiquitous computing and smart devices Wireless sensor networks, IPV6, Handoff Management, 6loWPAN and Network and Mobile Computing System.

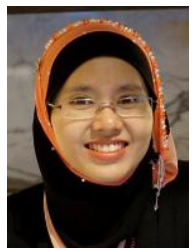

N. Effiyana Ghazali received the B.E. (2007) degree from Universiti Teknologi Malaysia. She received two M. E. degree, which is from Shibaura Institute of Technology (2010) and Universiti Teknologi Malaysia (2011). She received Ph.D (2016) from Universiti Teknologi Malaysia. Now she is working in Faculty of Electrical Engineering at Universiti Teknologi Malaysia who is currently active in research of Mobility Management, Sport Monitoring System, Ubiquitous Network, 5G and Handover Management.

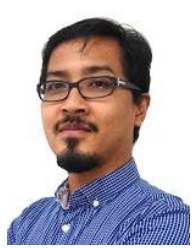

Kamaludin Mohamad Yusof received his B.Eng in 2000, MEE in 2012 from Universiti Teknologi Malaysia. He received Ph.D in 2014 from University of Essex, Essex. He is currently working with Faculty of Electrical Engineering, Universiti Teknologi Malaysia. His current research interests are in Signal Propagation, Ranging Estimation, Localisation, Wireless Sensor Network (WSN), Cognitive Radio (CR), Frequency Scanning, Software-Defined Radio (SDR), Networking, Software-Defined Network (SDN), Internet-of-Things (IoT), Crowd Sourcing and Data Mining.

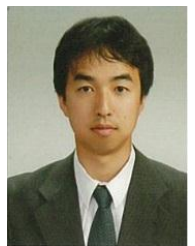

Hiroaki Morino received B.E, M.E. and Ph.D. degrees from the University of Tokyo in 1994, 1996 and 1999, respectively. He is currently an associate professor of Shibaura Institute of Technology, Japan. His research interests include the MAC protocols and routing protocols of wireless multihop networks, urgent information gathering and notification systems using wireless sensor networks, and reliable peer-to-peer video multicast networks. 